\title{
Call-Level Performance Sensitivity in Cellular Networks
}

\author{
Felipe A. Cruz-Pérez ${ }^{1}$, Genaro Hernández-Valdez ${ }^{2}$ and Andrés Rico-Páez ${ }^{1}$ \\ ${ }^{1}$ Electrical Engineering Department, CINVESTAV-IPN \\ ${ }^{2}$ Electronics Department, UAM-A \\ Mexico
}

\section{Introduction}

The development of analytically tractable teletraffic models for performance evaluation of mobile cellular networks under more realistic assumptions has been the concern of recent works (Corral-Ruíz et al. 2010; Fang a; 2005, Fang b; 2005; Kim \& Choi 2009; Pattaramalai, 2009; Rico-Páez et al., 2007; Rodríguez-Estrello et al., 2009; Rodríguez-Estrello et al., 2010; Wang \& Fan, 2007; Yeo \& Yun, 2002; Zeng et al., 2002). The general conclusion of those works is that, in order to capture the overall effects of cellular shape, cellular size, users' mobility patterns, wireless channel unreliability, handoff schemes, and characteristics of new applications, most of the time interval variables (i.e., those used for modeling time duration of different events in telecommunications - for example, cell dwell time, residual cell dwell time, unencumbered interruption time, unencumbered service time) need to be modeled as random variables with general distributions. In this research direction, phasetype distributions have got a lot of attention because of the possibility of using the theory of Markov processes ${ }^{1}$ (Fang, 1999, Christensen et al., 2004). Moreover, there have been major advances in fitting phase-type distributions to real data (Alfa \& Li, 2002). Among the phasetype probability distributions, the use of hyper-Erlang distribution is of special interest due to its universality property (i.e., it can be used to accurately approximate the behavior of any non negative random variable) and also because of the fact that it provides accurate description of real distributions of different time variables in mobile cellular networks (Fang, 1999; Corral-Ruíz et al., 2010; Yeo \& Yun, 2002).

When a probability distribution different to the negative exponential one is the best choice to fit the real distribution of a given time interval variable, not only its expected value but also its higher order moments are relevant. Nonetheless, the study of the effect of moments higher than the expected value has been largely ignored. The reason is twofold: 1) because of the relatively recent use of probability distributions different to the exponential one and 2) because the related works have been focused on developing mathematical models rather than numerically evaluating system performance.

In this chapter, the important task of identify and analyze the influence of moments higher than the expected value of both cell dwell time and unencumbered interruption time on

${ }^{1}$ Markovian properties are essential in generating tractable queuing models for mobile cellular networks. 
network performance is addressed. Specifically, in this chapter, system performance sensitivity to the first three standardized moments (i. e., expected value, coefficient of variation, and skewness) of both cell dwell time and unencumbered interruption time in cellular networks is investigated. These time interval variables are assumed to be phase-type distributed random variables. System performance is evaluated in terms of new call blocking, handoff failure, and forced call termination probabilities, carried traffic, handoff call arrival rate, and the mean channel holding time for new and handed off calls.

\section{System model description}

In this section, the basic concepts and general guidelines for the mathematical analysis developed in subsequent sections are given.

\subsection{Basic concepts}

In a mobile cellular network, radio links for communication are provided by base stations whose radio coverage defines a cell. Every time a mobile user whishes to initiate a call, the mobile terminal attempts to obtain a radio channel for the connection. If no channel is available, the call is blocked and cleared from the network. This is called a new call blocking. Nonetheless, if a channel is available, it is used for the connection, and released under any of the following situations: the call is successfully completed, the call is forced to terminate due to the wireless link unreliability, or the mobile user moves out of the cell. The channel holding time is defined as the amount of time that a call occupies a channel in a particular cell.

Moreover, when a mobile user moves from one cell to another during an ongoing communication, the call requires a new channel to be reserved in the new cell. This procedure of changing channels is called a handoff. If no channel is available in the new cell during the handoff, the call is said to be forced to terminate due to resource insufficiency. This phenomenon is called a handoff call blocking. New call blocking and forced call termination probabilities are being considered as important design parameters for evaluating the level of quality of service (QoS) offered by a wireless network. It has been observed that priority to handoff calls over new call initiation enables to improve the QoS. In a well-established cellular network and from the call forced termination point of view, handoff call blocking can be usually a negligible event (Boggia et al., 2005). Thus, the main cause of call forced termination is due to the unreliable nature of the wireless communication channel² (Boggia et al., 2005).

\subsection{Basic assumptions}

A homogeneous multi-cellular system with omni-directional antennas located at the centre of each cell is assumed; that is, the underlying processes and parameters for all cells within the cellular network are the same, so that all cells are statistically identical. Each cell has a

\footnotetext{
2 Physical link is said to be unreliable if the experienced signal to interference ratio (SIR) is lower in value than a minimum required value (SIR threshold) for more than a specified period of time (time threshold). During the course of a call, the physical link between base station and mobile station may suffer link unreliability due to propagation impairments such as multi-path fading, shadowing or path loss, and interference (Rodríguez-Estrello et al., 2010).
} 
maximum number $S$ of radio channels assigned to it and can therefore support at most $S$ calls simultaneously. Since a sudden forced termination during a call session will be more upsetting than a failure to connect, a fractional cutoff priority scheme is used to give handoff calls priority over new calls. For this purpose, a real number $N$ of channels in each cell is reserved for handoff prioritization (Vázquez-Ávila et al., 2006). As it has been widely accepted in the related literature (Orlik \& Rappaport, 1998; Lin et al., 1994), both the new call arrivals and handoff attempts are assumed to follow independent Poisson processes with mean arrival rate $\lambda_{n}$ and $\lambda_{h}$, respectively, per cell. Some other assumptions and definitions are presented in the Section 2.3.

\subsection{Definition of time interval variables}

In this section the different time interval variables involved in the teletraffic model of a mobile cellular network are defined.

First, the unencumbered service time per call $x_{s}$ (also known as the requested call holding time (Alfa and Li, 2002) or call holding duration (Rahman \& Alfa, 2009)) is the amount of time that the call would remain in progress if it experiences no forced termination. It has been widely accepted in the literature that the unencumbered service time can adequately be modeled by a negative exponentially distributed random variable (RV) (Lin et al., 1994; Hong \& Rappaport, 1986, Del Re et al., 1995). The RV used to represent this time is $\mathbf{X}_{s}$ and its mean value is $\mathrm{E}\left\{\mathbf{X}_{s}\right\}=1 / \mu$.

Now, cell dwell time or cell residence time $x_{d}(j)$ is defined as the time interval that a mobile station (MS) spends in the $j$-th (for $j=0,1, \ldots$ ) handed off cell irrespective of whether it is engaged in a call (or session) or not. The random variables (RVs) used to represent this time are $\mathbf{X}_{d}^{(j)}($ for $j=0,1, \ldots)$ and are assumed to be independent and identically generally phasetype distributed. For homogeneous cellular systems, this assumption has been widely accepted in the literature (Lin et al., 1994; Hong \& Rappaport, 1986, Del Re et al., 1995; Orlik \& Rappaport, 1998; Fang \& Chlamtac, 1999, Li \& Fang, 2008; Alfa \& Li, 2002; Rahman \& Alfa, 2009). $1 / \eta$ is the mean cell dwell time. In this Chapter, cell dwell time is modeled as a phase-type distributed RV.

The residual cell dwell time $x_{r}$ is defined as the time between the instant that a new call is initiated and the instant that the user is handed off to another cell. Notice that residual cell dwell time is only defined for new calls. The RV used to represent this time is $\mathbf{X}_{r}$. Thus, the probability density function (pdf) of $\mathbf{X}_{r}, f_{\mathbf{X}_{r}}(t)$, can be calculated in terms of $\mathbf{X}_{d}$ using the excess life theorem (Lin et al., 1994)

$$
f_{\mathbf{X}_{r}}(t)=\frac{1}{E\left[\mathbf{X}_{d}\right]}\left[1-F_{\mathbf{X}_{d}}(t)\right]
$$

where $E\left[\mathbf{X}_{d}\right]$ and $F_{\mathbf{X}_{d}}(t)$ are, respectively, the mean value and cumulative probability distribution function (CDF) of $\mathbf{X}_{d}$.

Finally, the mathematical model used to consider link unreliability is based on the proposed call interruption process proposed in (Rodríguez-Estrello et al., 2009). In (Rodríguez-Estrello et al., 2009), an interruption model and a potential associated time to this process, which is called "unencumbered call interruption time," is proposed. Unencumbered call interruption time $x_{i}(j)$ is defined as the period of time from the epoch the mobile terminal establish a link 
with the $j$-th handed-off cell (for $j=0,1,2, \ldots$ ) until the instant the call would be interrupted due to the wireless link unreliability assuming that the mobile terminal has neither successfully completed his call nor has been handed off to another cell. The RVs used to represent this time are $\mathbf{X}_{i}(j)$ (for $j=0,1,2, \ldots$ ). These RVs are assumed to be independent and phase-type distributed (Rodríguez-Estrello et al., 2009; Rodríguez-Estrello et al., 2010).

Relationships between the different time interval variables defined in this section are illustrated in Fig. 1. Specifically, Fig. 1 shows the time diagram for a forced terminated call due to link unreliability.

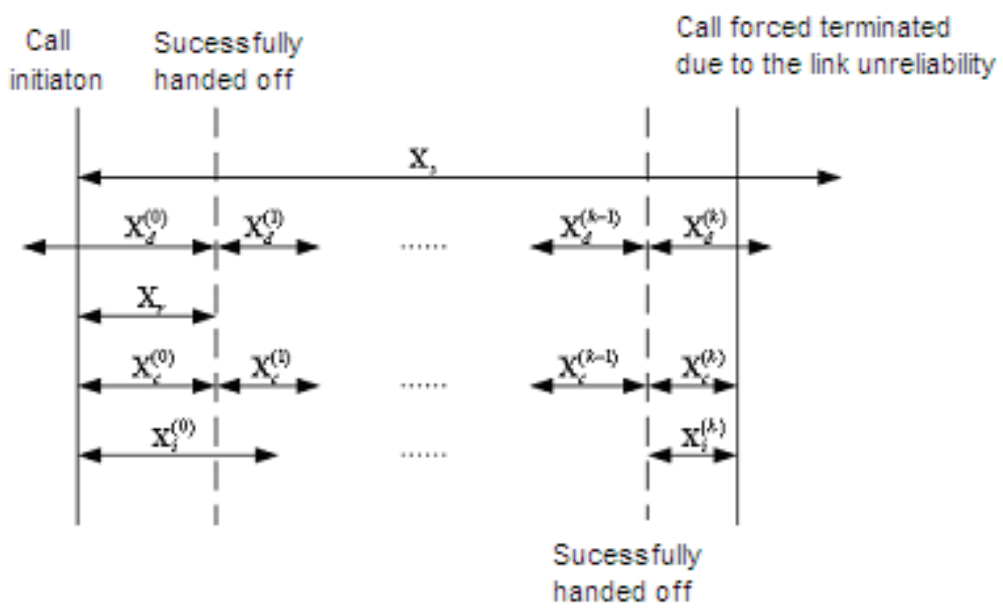

Fig. 1. Time diagram for a forced terminated call due to link unreliability.

\section{Teletraffic analysis}

In this section, the teletraffic analytical method for system-level evaluation of mobile cellular networks is presented. To avoid analytical complexity and for an easy interpretation of our numerical results, exponentially distributed unencumbered interruption time and hyperErlang distributed cell dwell time are considered. Nonetheless, numerical results for the following cases are also presented and discussed in Section 5 (Performance Evaluation): 1) Cell dwell time hyper-exponential distributed and unencumbered interruption time exponential distributed, 2) Cell dwell time exponential distributed and unencumbered interruption time hyper-Erlang distributed, 3) Cell dwell time exponential distributed and unencumbered interruption time hyper-exponential distributed.

\subsection{Residual cell dwell time characterization}

The methodology by which we can model the residual cell dwell time distribution is described in this section.

Suppose that cell dwell time follows an $m^{(h)}$-th order hyper-Erlang distribution function with parameters $\alpha_{i}(h), u_{i}(h)$, and $\eta_{i}(h)$ (for $\left.i=1,2, \ldots, m^{(h)}\right)$. Let us represent by $\alpha_{i}(h)$ the probability of 
choosing the phase $i$ (for $i=1,2, \ldots, m^{(h)}$ ). Thus, the pdf of cell dwell time can be written as follows

$$
f_{\mathbf{X}_{d}}(t)=\sum_{i=1}^{m^{(h)}} \alpha_{i}^{(h)} \frac{\left(\eta_{i}^{(h)}\right)^{u_{i}^{(h)}} t^{u_{i}^{(h)}-1}}{\left(u_{i}^{(h)}-1\right) !} e^{-\eta_{i}^{(h)} t} ; \eta_{i}^{(h)}>0, \quad t \geq 0,0 \leq \alpha_{i}^{(h)} \leq 1, \sum_{i=1}^{m^{(h)}} \alpha_{i}^{(h)}=1
$$

where $u_{i}^{(h)}$ is a positive integer and $\eta_{i}(h)$ is a positive constant. Note that the hyper-Erlang distribution is a mixture of $m^{(h)}$ different Erlang distributions, and each of them has a shape parameter $u_{i}(h)$ and a rate parameter $\eta_{i}(h)$. The rate parameter is related to the mean cell dwell time as follows: $\eta_{i}(h)=\eta u_{i}(h)$ (Fang, 1999). The value $\alpha_{i}(h)$ represents the weight of each Erlang distribution. Using (1), the pdf of residual cell dwell time can be computed as follows

$$
f_{\mathbf{X}_{r}}(t)=\frac{1}{\sum_{i=1}^{m^{(h)}} \frac{\alpha_{i}^{(h)} u_{i}^{(h)}}{\eta_{i}^{(h)}} \sum_{i=1}^{m^{(h)}} \sum_{j=0}^{(h)}-1} \alpha_{i}^{(h)} \frac{\left(\eta_{i}^{(h)} t\right)^{j}}{j !} e^{-\eta_{i}^{(h)} t}
$$

It is straightforward to show that the probability distribution function (pdf) of residual cell dwell time can be rewritten in the following compact form

$$
f_{\mathbf{X}_{r}}(t)=\sum_{i=1}^{m^{(n)}} \alpha_{i}^{(n)} \frac{\left(\eta_{i}^{(n)}\right)^{u_{i}^{(n)}} t^{u_{i}^{(n)}-1}}{\left(u_{i}^{(n)}-1\right) !} e^{-\eta_{i}^{(n)} t}
$$

where

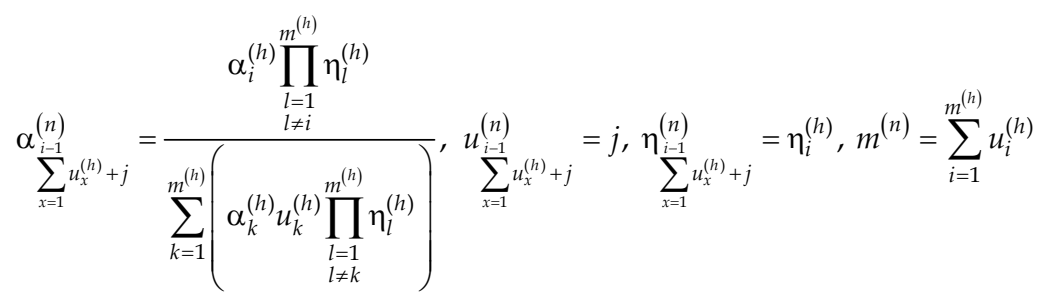

for $i=1,2, \ldots, m^{(h)}, j=1,2, \ldots, u_{i}^{(h)}$.

From (4), it is not difficult to notice that residual cell dwell time is an $m^{(n)}$-th order hyperErlang random variable with shape and rate parameters $u_{i}(n)$ and $\eta_{i}(n)$ (for $\left.i=1,2, \ldots, m^{(n)}\right)$. Also, $\mathrm{a}_{i}(n)$ represents the probability of choosing the phase $i$ (for $\left.i=1,2, \ldots, m^{(n)}\right)$. The diagram of phases and stages of cell dwell time and residual cell dwell time is shown in Fig. 2. In Fig. 2, $y=\{n\}$ represents the case when residual cell dwell time is considered, while $y=\{h\}$ represents the case when cell dwell time is considered. 


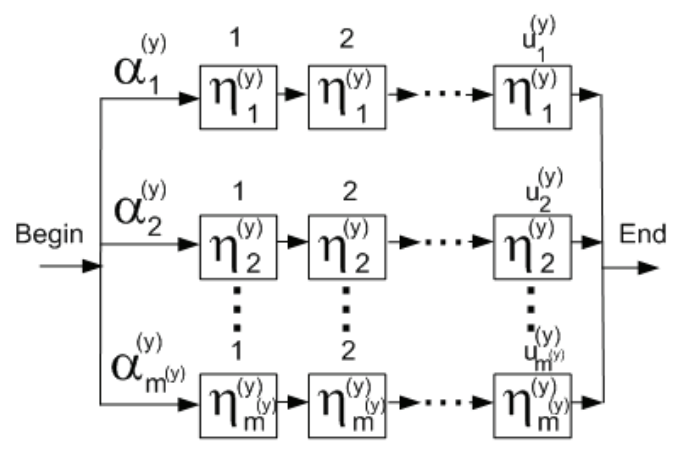

Fig. 2. Diagram of phases and stages of the probability distribution of residual cell dwell time $(y=\{n\})$ and cell dwell time $(y=\{h\})$.

\subsection{Queuing formulation}

In the context of a wireless network, each cell may be modelled as a queuing system where new and handoff arrivals correspond to connection requests or call origination, the departures correspond to disconnection due to call termination, force termination due to wireless link unreliability, or handoff throughout adjacent cells. The servers represent the available channels, whereas the clients represent the active mobile terminals. Since a homogenous case is assumed where all the cells in the service area are statistically identical, the overall system performance can be analyzed by focusing on only one given cell. In this Chapter, we analyze system performance by following the approach proposed in (RicoPáez, et al., 2007; Rico-Páez et al., 2009; Rico-Páez et al., 2010) to capture the general distributions for both cell dwell time and unencumbered call interruption time. In this section, the special case when UIT is exponential distributed and CDT is hyper-Erlang distributed is considered. The mean value of UIT is $1 / \gamma$. For modelling this system through a multidimensional birth and death process, a total number of $\sum_{x=1}^{m(n)} u_{x}^{(n)}+\sum_{x=1}^{m^{(h)}} u_{x}^{(h)}$ state variables are needed. Let us define $k_{\sum_{x=1}^{(y)} u_{x}^{(y)}+j}$ as the number of users in stage $i$ and phase $j$ of residual cell dwell time $(y=\{n\})$ and cell dwell time $(y=\{h\})$.

To simplify mathematical notation, let us define the vector $\mathbf{K}^{(y)}$ as follows

$$
\mathbf{K}^{(y)}=\left[k_{1}^{(y)}, k_{2}^{(y)}, \ldots, k^{(y)} \sum_{i=1}^{m^{(y)}} u_{i}^{(y)}\right]
$$

Also, let us define the vector $\mathbf{e}_{i}^{(y)}$ as a unit vector of dimension $m^{(y)}$ whose all entries are 0 except the $i$-th one which is 1 (for $i=1,2, \ldots, \Sigma_{i=1}^{m^{(y)}} u_{i}^{(y)}$ ). Let us define the current state of the analyzed cell as the vector $\left[\mathbf{K}^{(n)}, \mathbf{K}^{(h)}\right.$ ]. Table I provides the rules that determine transition rates to the different successor states (shown in the second column). As stated before, we assume that all the cells are probabilistically equivalent. That is, the new call arrival rate in each cell is equal, and the rate at which mobiles enter a given cell is equal to the rate at which they interrupt its connection (due to either a handed off call event or link unreliability) to that cell. Thus, equating rate out to rate in for each state, the statisticalequilibrium state equations are given by (Cooper, 1990): 


$$
\begin{aligned}
& P\left(\left[\overline{\mathbf{K}^{(n)}}, \overline{\mathbf{K}^{(h)}}\right]\right)=\frac{g_{a}^{(n)}+g_{b}^{(n)}+g_{c}^{(n)}+g_{d}^{(n)}+g_{a}^{(h)}+g_{b}^{(h)}+g_{c}^{(h)}+g_{d}^{(h)}}{\sum_{i=1}^{m^{(n)}} a_{i}^{(n)}\left(\left[\overline{\mathbf{K}^{(n)}}, \overline{\mathbf{K}^{(h)}}\right]\right)+\sum_{i=1}^{m^{(n)}} \sum_{j=1}^{(n)} \sum_{i=1}^{(n)}\left(\left[\overline{\left.u_{x}^{(n)}-1\right)+j}\left(\overline{\mathbf{K}^{(n)}}, \overline{\mathbf{K}^{(h)}}\right]\right)+\sum_{i=1}^{m^{(n)}} c_{i}^{(n)}\left(\left[\overline{\mathbf{K}^{(n)}}, \overline{\mathbf{K}^{(h)}}\right]\right)\right.} \\
& +\sum_{i=1}^{m^{(n)}} \sum_{j=1}^{(n)} d_{x=1}^{(n)}\left(u_{x}^{(n)}-1\right)+j\left(\left[\overrightarrow{\mathbf{K}^{(n)}}, \overrightarrow{\mathbf{K}^{(h)}}\right]\right)+\sum_{i=1}^{m^{(h)}} \sum_{j=1}^{(h)} \sum_{x=1}^{(h)}\left(u_{x}^{(h)}-1\right)+j\left(\left[\overrightarrow{\mathbf{K}^{(n)}}, \overrightarrow{\mathbf{K}^{(h)}}\right]\right) \\
& +\sum_{i=1}^{m^{(h)}} a_{i}^{(h)}\left(\left[\overline{\mathbf{K}^{(n)}}, \overline{\mathbf{K}^{(h)}}\right]\right)+\sum_{i=1}^{m^{(h)}} c_{i}^{(h)}\left(\left[\overline{\mathbf{K}^{(n)}}, \overline{\mathbf{K}^{(h)}}\right]\right)+\sum_{i=1}^{m^{(h)}} \sum_{j=1}^{(h)} \sum_{x=1}^{(h)}\left(u_{x}^{(h)}-1\right)+j\left(\left[\overline{\mathbf{K}^{(n)}}, \overline{\mathbf{K}^{(h)}}\right]\right) \\
& \left\{\left(\begin{array}{l}
0 \leq k_{i}^{(n)} \leq S-N \text { for } i=1,2, \ldots, \sum_{x=1}^{m^{(n)}} u_{x}^{(n)} \\
0 \leq k_{i}^{(h)} \leq S \text { for } i=1,2, \ldots, \sum_{x=1}^{m^{(h)}} u_{x}^{(h)}
\end{array}\right) \cap\left(\sum_{i=1}^{\sum_{x=1}^{m^{(n)}} u_{x}^{(n)} k_{i}^{(n)}+\sum_{i=1}^{m_{x=1}^{(h)}} u_{x}^{(h)} k_{i}^{(h)} \leq S}\right)\right\}
\end{aligned}
$$

where

$$
\begin{aligned}
& g_{a}^{(n)}=\sum_{i=1}^{m^{(n)}}\left[a_{i}^{(n)}\left(\left[\overrightarrow{\mathbf{K}^{(n)}}-\overrightarrow{\mathbf{e}_{i-1}^{(n)} u_{x}^{(n)}+1}, \overline{\mathbf{K}^{(n)}}\right]\right) P\left(\left[\overrightarrow{\mathbf{K}^{(n)}}-\overrightarrow{\mathbf{e}_{i-1}^{(n)} u_{x}^{(n)}+1}, \overrightarrow{\mathbf{K}^{(h)}}\right]\right)\right] \\
& g_{b}^{(n)}=\sum_{i=1}^{m^{(n)}} \sum_{j=1}^{(n)}-1\left[b_{x=1}^{(n)}\left(u_{x}^{(n)}-1\right)+j\left(\left[\overline{\mathbf{K}^{(n)}}+\overline{\mathbf{e}_{i-1}^{(n)}}-\overline{\sum_{x=1}^{(n)}+j}-\overline{\mathbf{e}_{i-1}^{(n)} u_{x=1}^{(n)}+j+1}, \overline{\mathbf{K}^{(h)}}\right]\right) P\left(\left[\overline{\mathbf{K}^{(n)}}+\overline{\mathbf{e}_{i-1}^{(n)} u_{x}^{(n)}+j}-\overline{\mathbf{e}_{i-1}^{(n)}}, \overline{\sum_{x=1}^{(n)} u_{x}^{(n)}+j+1}\right]\right)\right] \\
& g_{c}^{(n)}=\sum_{i=1}^{m^{(n)}}\left[c_{i}^{(n)}\left(\left[\overrightarrow{\mathbf{K}^{(n)}}+\overrightarrow{\mathbf{e}_{i}^{(n)}, u_{x}^{(n)}}, \overrightarrow{\mathbf{K}^{(h)}}\right]\right) P\left(\left[\overrightarrow{\mathbf{K}^{(n)}}+\overrightarrow{\mathbf{e}_{i}^{(n)},}, \overrightarrow{\sum_{x=1}^{(n)} u_{x}^{(n)}}\right]\right)\right] \\
& g_{d}^{(n)}=\sum_{i=1}^{m^{(n)}} \sum_{j=1}^{u_{i}^{(n)}-1}\left[d_{i-1}^{(n)}\left(u_{x=1}^{(n)}-1\right)+j\left(\left[\overrightarrow{\mathbf{K}^{(n)}}+\overrightarrow{\mathbf{e}_{i-1}^{(n)}}, \overrightarrow{\sum_{x=1}^{(n)} u_{x}^{(n)}+j}\right]\right) P\left(\left[\overrightarrow{\mathbf{K}^{(n)}}+\overrightarrow{\mathbf{e}_{i-1}^{(n)} u_{x}^{(n)}+j}, \overrightarrow{\mathbf{K}^{(h)}}\right]\right)\right] \\
& g_{a}^{(h)}=\sum_{i=1}^{m^{(h)}}\left[a_{i}^{(h)}\left(\left[\overrightarrow{\mathbf{K}^{(n)}}, \overrightarrow{\mathbf{K}^{(h)}}-\overrightarrow{\mathbf{e}_{i-1}^{(h)} u_{x}^{(h)}+1}\right]\right) P\left(\left[\overrightarrow{\mathbf{K}^{(n)}}, \overrightarrow{\mathbf{K}^{(h)}}-\overrightarrow{\mathbf{e}_{i-1}^{(h)}}\right]\right)\right]
\end{aligned}
$$




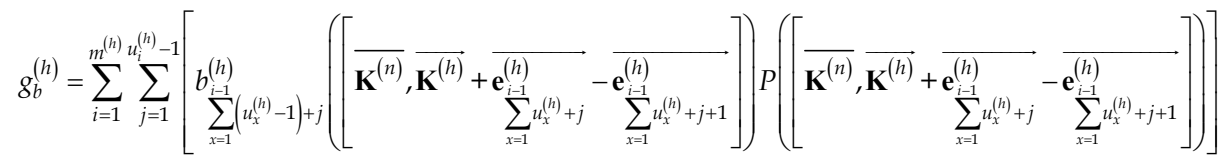

$$
\begin{aligned}
& \left.g_{c}^{(h)}=\sum_{i=1}^{m^{(h)}}\left[c_{i}^{(h)}\left(\left[\overrightarrow{\mathbf{K}^{(n)}}, \overrightarrow{\mathbf{K}^{(h)}}+\overrightarrow{\mathbf{e}_{i}^{(h)}}\right]\right) P\left(\left[\overrightarrow{\sum_{x=1}^{(h)}}\right] \overrightarrow{\mathbf{K}_{x}^{(n)}}, \overrightarrow{\mathbf{K}^{(h)}}+\overrightarrow{\mathbf{e}_{i}^{(h)} u_{x}^{(h)}}\right]\right)\right] \\
& g_{d}^{(h)}=\sum_{i=1}^{m^{(h)}} \sum_{j=1}^{(h)}\left[\sum_{x=1}^{(h)}\left[u_{x}^{(h)}-1\right)+j\left(\left[\overrightarrow{\mathbf{K}^{(n)}}, \overrightarrow{\mathbf{K}^{(h)}}+\overrightarrow{\mathbf{e}_{i-1}^{(h)}}\right]\right) P\left(\left[\overrightarrow{\mathbf{K}_{x=1}^{(h)}}, \overrightarrow{\mathbf{K}^{(h)}}+\overrightarrow{\mathbf{e}_{i-1}^{(h)}}\right]\right)\right]
\end{aligned}
$$

Of course, the probabilities must satisfy the normalization equation given by (15).

$$
\begin{aligned}
& \sum_{k_{1}^{(n)}=0}^{S-N} \ldots \sum_{\substack{k^{(n)} \\
m_{\sum}^{(n)} u_{x}^{(n)}}}^{S-N} \sum_{\substack{(h) \\
k_{1}^{(h)}=0}}^{S} \ldots \sum_{\substack{k^{(h)} \\
\sum_{\begin{subarray}{c}{(h) \\
x=1} }}^{(h)}}\end{subarray}}^{S} P\left(\left[\overrightarrow{\mathbf{K}^{(n)}}, \overrightarrow{\mathbf{K}^{(h)}}\right]\right)=1
\end{aligned}
$$

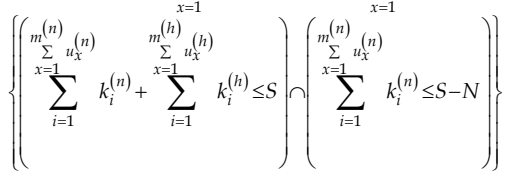

The corresponding steady state probabilities are calculated by means of the Gauss-Seidel Method (Cooper, 1990).

Let us assume that the channels reserved for handoff prioritization are given by $N(n)=N$ and $N(h)=0$, respectively, and the total number of channels is $S$. Transition rates shown in Table I are given by (for $y=\{n, h\}$ )

$$
\begin{aligned}
& a_{i}^{(y)}\left(\left[\mathbf{K}^{(n)}, \mathbf{K}^{(h)}\right]\right)=\left\{\begin{array}{l}
\mathbf{a}_{i}^{(y)} \lambda^{(y)} ; \sum_{l=1}^{\sum_{x=1}^{m(n)} u_{x}^{(n)}} k_{l}^{(n)}+\sum_{l=1}^{\sum_{x=1}^{m^{(h)}} u_{x}^{(h)}} k_{l}^{(h)}<S-N^{(y)} \cap k_{i-1}^{(y)} u_{x}^{(y)}+1 \\
0 \quad ; \text { otherwise }
\end{array}\right. \\
& b_{x=1}^{(y)}\left(u_{x}^{(y)}-1\right)+j
\end{aligned}
$$




$$
\begin{aligned}
& c_{i}^{(y)}\left(\left[\mathbf{K}^{(n)}, \mathbf{K}^{(h)}\right]\right)= \begin{cases}k_{x=1}^{(y)}\left(\mu+\eta_{i}^{(y)}+\gamma\right) ; & \sum_{l=1}^{m_{i}^{(n)}} k_{l}^{(n)}+\sum_{l=1}^{(n)} k_{l}^{(y)} \leq S \cap k_{x=1}^{(y)} \sum_{x=1}^{m^{(h)} u_{x}^{(y)}}>0 \\
0 \quad \text { otherwise } & \end{cases}
\end{aligned}
$$

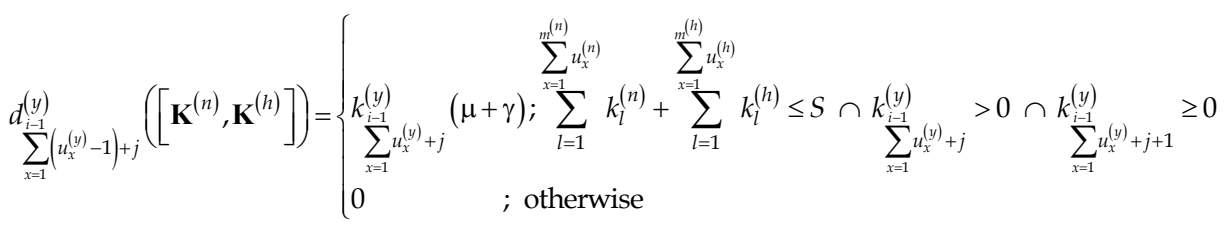

The new call blocking $\left(P^{(n)}\right)$ and handoff failure $\left(P^{(h)}\right)$ probabilities can be computed using (20).

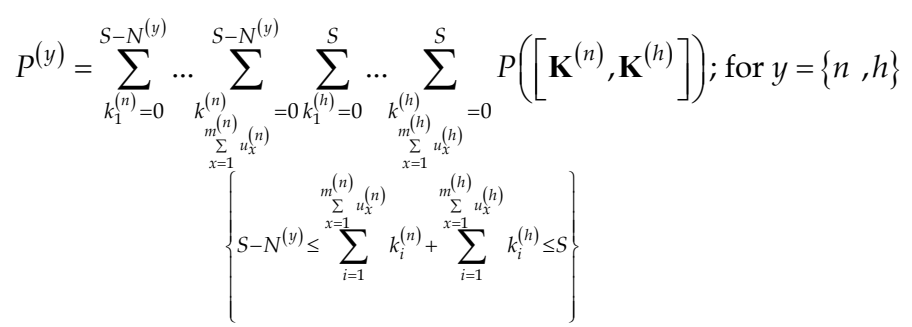

Finally, the carried traffic $\left(a_{c}\right)$ can be computed as follows

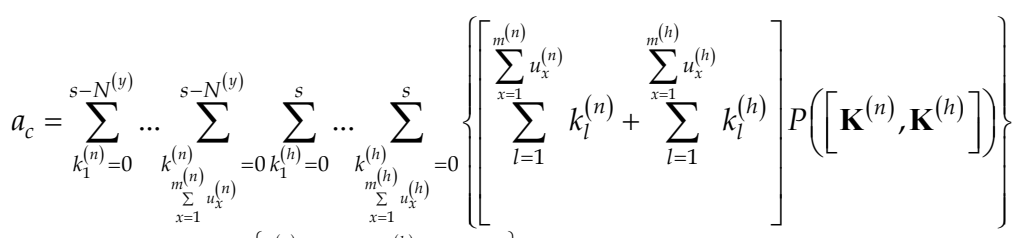

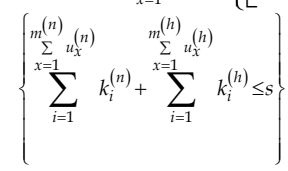

Call forced termination probability can be calculated using the methodology developed in Section 4, while the handoff call attempt rate is calculated iteratively as explained in (Lin et al., 1994).

\section{Forced termination probability}

Forced termination may result from either link unreliability or due to handoff failure. In general, a dropped call suffers $j(j=0,1,2, \ldots)$ successful handoffs and one forced interruption (due to either a handoff failure or link unreliability) before it is forced terminated. Thus, the forced termination probability in cell $j$ can be expressed as follows 


\begin{tabular}{|c|c|c|}
\hline Event & Successor State & Rate \\
\hline $\begin{array}{l}\text { Call enters first phase of stage } i \text { of } \\
\mathbf{X}_{r}\left(i=1,2, \ldots, m^{(n)}\right)\end{array}$ & 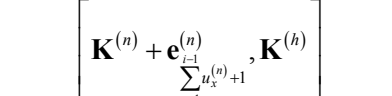 & $a_{i}^{(n)}\left(\left[\mathbf{K}^{(n)}, \mathbf{K}^{(h)}\right]\right)$ \\
\hline $\begin{array}{l}\text { Call leaves phase } j \text { of stage } i \text { and } \\
\text { enters phase } j+1 \text { of stage } i \text { of } \mathbf{X}_{r}(i=1 \text {, } \\
\left.2, . ., m^{(n)}\right),\left(j=1,2, \ldots, u_{i}^{(n)}-1\right)\end{array}$ & {$\left[\mathbf{K}^{(n)}-\mathbf{e}_{i=1}^{(n)} \sum_{x=1}^{(n)} u_{x}^{(n)}+\mathbf{e}_{i=1}^{(n)} \sum_{x=1}^{(n)} u_{x}^{(n)+j+1}\right]$} & $\sum_{x=1}^{(n)}\left(u_{x}^{(n)-1)+j}\right)\left(\left[\mathbf{K}^{(n)}, \mathbf{K}^{(h)}\right]\right)$ \\
\hline $\begin{array}{l}\text { Call leaves last phase of stage } i \text { of } \mathbf{X}_{r} \\
\left(i=1,2, \ldots, m^{(n)}\right)\end{array}$ & $\mathbf{K}^{(n)}-\mathbf{e}_{\sum_{i=1}^{(n)} u_{x}^{(n)}}^{(n)}, \mathbf{K}^{(h)}$ & $c_{i}^{(n)}\left(\left[\mathbf{K}^{(n)}, \mathbf{K}^{(h)}\right]\right)$ \\
\hline $\begin{array}{l}\text { Call leaves phase of stage } i \text { of } \mathbf{X}_{r} \\
\left(i=1,2, \ldots, m^{(n)}\right)\end{array}$ & $\mathbf{K}^{(n)}-\mathbf{e}_{\sum_{x=1}^{(n)} u_{x}^{(n)}+j}, \mathbf{K}^{(h)}$ & 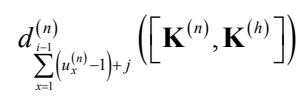 \\
\hline $\begin{array}{l}\text { Call enters first phase of stage } i \text { of } \\
\mathbf{X}_{d}\left(i=1,2, \ldots, m^{(h)}\right)\end{array}$ & $\mathbf{K}^{(n)}, \mathbf{K}^{(h)}+\mathbf{e}_{i=1}^{(h)} u_{i=1}^{(h)} u_{x}^{(h)}$ & $a_{i}^{(h)}\left(\left[\mathbf{K}^{(n)}, \mathbf{K}^{(h)}\right]\right)$ \\
\hline $\begin{array}{l}\text { Call leaves phase } j \text { of stage } i \text { and } \\
\text { enters phase } j+1 \text { of stage } i \text { of } \mathbf{X}_{d} \\
\left(i=1,2, \ldots, m^{(h)}\right)\left(j=1,2, \ldots, u_{i}^{(h)}-1\right)\end{array}$ & 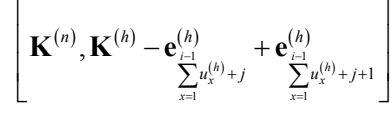 & $\sum_{x=1}^{(h)}\left(u_{x}^{(h)-1)+j}\left(\left[\mathbf{K}^{(n)}, \mathbf{K}^{(h)}\right]\right)\right.$ \\
\hline $\begin{array}{l}\text { Call leaves last phase of stage } i \text { of } \mathbf{X}_{d} \\
\left(i=1,2, \ldots, m^{(h)}\right)\end{array}$ & $\left.\mathbf{K}^{(n)}, \mathbf{K}^{(h)}-\mathbf{e}_{\sum_{x=1}^{(h)} u_{x}^{(h)}}\right]$ & $c_{i}^{(h)}\left(\left[\mathbf{K}^{(n)}, \mathbf{K}^{(h)}\right]\right)$ \\
\hline $\begin{array}{l}\text { Call leaves phase of stage } i \text { of } \mathbf{X}_{d} \\
\left(i=1,2, \ldots, m^{(n)}\right)\end{array}$ & $\mathbf{K}^{(n)}, \mathbf{K}^{(h)}-\mathbf{e}_{\sum_{x=1}^{(h)} u_{x}^{(h)}+j}$ & $d_{\sum_{x=1}^{(h)}\left(u_{x}^{(h)}-1\right)+j}\left(\left[\mathbf{K}^{(n)}, \mathbf{K}^{(h)}\right]\right)$ \\
\hline
\end{tabular}

Table 1. Transition rules for the case when the cell residence time is hyper-Erlang distributed and unencumbered interruption time is negative exponential distributed. 


$$
P_{f t}^{(j)}=\left\{\begin{array}{l}
P\left(\mathbf{X}_{i} \leq \min \left(\mathbf{X}_{s}, \mathbf{X}_{r}\right)\right)+P\left(\mathbf{X}_{r} \leq \min \left(\mathbf{X}_{s}, \mathbf{X}_{i}\right)\right) P_{h} \quad ; j=0 \\
P\left(\mathbf{X}_{r} \leq \min \left(\mathbf{X}_{s}, \mathbf{X}_{i}\right)\right)\left(1-P_{h}\right)\left[P\left(\mathbf{X}_{i} \leq \min \left(\mathbf{X}_{s}, \mathbf{X}_{d}\right)\right)+P\left(\mathbf{X}_{d} \leq \min \left(\mathbf{X}_{s}, \mathbf{X}_{i}\right)\right) P_{h}\right] ; j=1 \\
P\left(\mathbf{X}_{r} \leq \min \left(\mathbf{X}_{s}, \mathbf{X}_{i}\right)\right) P\left(\mathbf{X}_{d} \leq \min \left(\mathbf{X}_{s}, \mathbf{X}_{i}\right)\right)^{j-1}\left(1-P_{h}\right)^{j} \times \\
{\left[P\left(\mathbf{X}_{i} \leq \min \left(\mathbf{X}_{s}, \mathbf{X}_{d}\right)\right)+P\left(\mathbf{X}_{d} \leq \min \left(\mathbf{X}_{s}, \mathbf{X}_{i}\right)\right) P_{h}\right] ; j>1}
\end{array}\right.
$$

where $P_{h}$ represents the handoff failure probability. $P\left(\mathbf{X}_{i} \leq \min \left(\mathbf{X}_{s}, \mathbf{X}_{r}\right)\right.$ and $P\left(\mathbf{X}_{i} \leq \min \left(\mathbf{X}_{s}, \mathbf{X}_{d}\right)\right.$ represent interruption probabilities due to link unreliability for new and handoff calls, respectively. Function $\min (\cdot, \cdot)$ returns the minimum of two random variables.

The call forced termination can happen in any cell. Therefore, using the total probability theorem, the forced termination probability can be computed as follows

$$
\begin{aligned}
P_{f t}= & \sum_{j=0}^{\infty} P_{f t}^{(j)} \\
= & P\left(\mathbf{X}_{i} \leq \min \left(\mathbf{X}_{s}, \mathbf{X}_{r}\right)\right)+P\left(\mathbf{X}_{r} \leq \min \left(\mathbf{X}_{s}, \mathbf{X}_{i}\right)\right) P_{h}+P\left(\mathbf{X}_{r} \leq \min \left(\mathbf{X}_{s}, \mathbf{X}_{i}\right)\right)\left(1-P_{h}\right) \times \\
& {\left[P\left(\mathbf{X}_{i} \leq \min \left(\mathbf{X}_{s}, \mathbf{X}_{d}\right)\right)+P\left(\mathbf{X}_{d} \leq \min \left(\mathbf{X}_{s}, \mathbf{X}_{i}\right)\right) P_{h}\right]\left[\frac{1}{1-P\left(\mathbf{X}_{d} \leq \min \left(\mathbf{X}_{s}, \mathbf{X}_{i}\right)\right)\left(1-P_{h}\right)}\right] }
\end{aligned}
$$

Let us define $\left.\mathbf{Z}_{s}(w)=\min \left(\mathbf{X}_{s}, \mathbf{X}_{w}\right)\right]$ with $w=\{r, i, d\}$. Notice that $\mathbf{Z}_{s}^{(w)}$ are non-negative RVs. Thus, the different probabilities in (23) can be calculated by using the following relationship (which uses the well known residual theorem) between two non-negative independent RVs (Fang a, 2005; Fang b, 2005)

$$
\begin{aligned}
P\left(\mathbf{X}_{w} \leq \mathbf{Z}_{s}^{(w)}\right) & =\frac{1}{2 \pi \mathrm{i}} \int_{\sigma-\mathrm{i} \infty}^{\sigma+\mathrm{i} \infty} \frac{f_{\mathbf{X}_{w}}^{*}(s)}{s} f_{\mathbf{Z}_{s}^{(w)}}^{*}(-s) d s \\
& =-\sum_{p \in \sigma_{P}} \operatorname{Res}_{s=p}\left[\frac{f_{\mathbf{X}_{w}}^{*}(s)}{s} f_{\mathbf{Z}_{s}^{(w)}}^{*}(-s)\right]
\end{aligned}
$$

where $f_{\mathbf{X}_{w}}^{*}(s)$ and $f_{\mathbf{Z}_{s}^{(w)}}^{*}(s)$ represent, respectively, the Laplace transform of $\mathbf{X}_{w}$ and $\mathbf{Z}_{s}^{(w)}$ with $w=\{r, i, d\} . \sigma_{P}$ is the set of poles of $f_{\mathbf{Z}_{s}^{(w)}}^{*}(-s)$. Equation (24) applies when the pdfs of $\mathbf{X}_{w}$ and $\mathbf{Z}_{s}(w)$ are proper rational functions (Fang, 2005). This is the situation of the different cases studied in this paper.

\section{Performance Evaluation}

The goal of the numerical evaluations presented in this section is to understand and analyze the influence of standardized moments higher than the expected value of both cell dwell time (CDT) and unencumbered interruption time (UIT) on the performance of mobile 
cellular networks. At least otherwise stated, in numerical evaluations it is assumed that mean service time is $1 / \mu=180 \mathrm{~s}$, total number of channels per cell $S=8$, offered traffic equal to 4.4 Erlangs per cell, and total number of channels reserved for handoff prioritization $N^{(n)}=1$. Figs. 3, 4, and $5(6,7$, and 8) show, respectively, forced termination probability, blocking probability, and carried traffic as function of both skewness and coefficient of variation of the unencumbered call interruption time (cell dwell time). Figs. 3, 4, and 5 show numerical results for the particular case when UIT is either hyper-Erlang or hyper-exponential distributed and CDT is exponential distributed. On the other hand, Figs. 6, 7, and 8 show numerical results for the particular case when CDT is either hyper-Erlang or hyperexponential distributed and UIT is exponential distributed. In Figs. 6-8, for the sake of comparison, two different values of the mean of the cell dwell time are considered: 100s (high mobility scenario) and $900 \mathrm{~s}$ (low mobility scenario). Also, two different values of the mean of the unencumbered call interruption time are considered in Figs. 3-5: $1500 \mathrm{~s}$ (low reliability scenario) and $5000 \mathrm{~s}$ (high reliability scenario) ${ }^{3}$.

\subsection{Influence of unencumbered interruption time statistics on system performance}

In this Section, the influence of the expected value, coefficient of variation, and skewness of unencumbered interruption time on system performance is investigated.

From Fig. 3 it is observed that as the mean value of the UIT decreases the forced termination probability increases, indicating a detrimental effect of channel unreliability on system performance (remember that physically, the mean value of UIT represents a direct measure of link reliability). On the contrary, as Fig. 4 shows, the blocking probability decreases as link unreliability increases (i.e., when mean unencumbered call interruption time decreases), indicating a positive effect of channel unreliability on system performance. This behavior can be explained as follows. As link unreliability increases, more ongoing calls are forced to terminate, consequently, more resources are available for new calls decreasing, in this way, new call blocking probability.

Figs. 3 and 4 also show that, irrespective of the value of skewness, forced termination probability increases and new call blocking probability decreases as CoV of UIT increases. This behavior can be explained as follows. First, note that as the $\mathrm{CoV}$ increases, the variability of the UIT increases, thus the probability that UIT takes smaller values increases and, consequently, more calls are interrupted due to link unreliability. This fact contributes to both increase forced termination probability and decrease new call blocking probability. Furthermore, from Figs. 3 and 4 it is rather interesting to note that, for low values of skewness (say, less that 20), forced termination probability significantly increases and new call blocking probability decreases as $\mathrm{CoV}$ increases. For instance, Figs. 3 and 4 shows that, for the low reliability scenario, skewness equals 2, and UIT Hyper-Erlang distributed, the forced termination probability increases around $700 \%$ and new call blocking probability decreases $67 \%$ as the CoV of UIT changes from 1 to 20 . Notice that the scenario where skewness equals 2 and $\mathrm{CoV}$ equals 1 corresponds to the case when UIT is negative

\footnotetext{
${ }^{3}$ Please note that both values of the mean of unencumbered call interruption time are significantly greater than the mean of cell dwell time. The reason of this is that communication systems are commonly designed to be reliable, thus mean unencumbered call interruption time should be typically greater than mean service time and mean cell dwell time.
} 
exponential distributed. Finally, from Fig. 3 (Fig. 4) observe that forced termination (new call blocking) probability is a monotonically decreasing (increasing) function of skewness. On the other hand, Fig. 5 shows that the carried traffic is an increasing function of both the skewness and mean value of UIT. Also, Fig. 5 shows that, for values of skewness smaller than around 30, carried traffic decreases as $\mathrm{CoV}$ of UIT increases. These observations indicate a detrimental effect of channel unreliability on carried traffic. Moreover, it is interesting to note that, for values of skewness grater than around 30 and for the same mean value and type of distribution of UIT, the carried traffic is almost insensitive to the CoV of UIT. The reason is as follows. Consider that the mean value, $\mathrm{CoV}$ and distribution type of UIT remain without change. Then, as the skewness of UIT increases, the tail on the right side of the UIT distribution function becomes longer (that is, the probability that UIT takes higher values increases and, consequently, less calls are interrupted due to link unreliability). In this manner, the influence of skewness on forced termination probability becomes negligible. At the same time, because of link unreliability is not considered to accept a call, the blocking probability is not sensitive to changes on neither skewness nor $\mathrm{CoV}$ of UIT statistics. As the carried traffic directly depends on both blocking and forced termination probabilities, the combined effect of these two facts lead us to the behavior observed in Fig. 5.

An interesting observation on the results illustrated in Figs. 3-5 is that, for the same scenario, skewness and $\mathrm{CoV}$, there exists a non-negligible difference between the values taken by the different performance metrics when UIT is modeled as Hyper-Erlang and Hyperexponential distributed random variable. Thus, it is evident that not only the expected value but also moments of higher order and the distribution model used to characterize UIT are relevant on system performance.

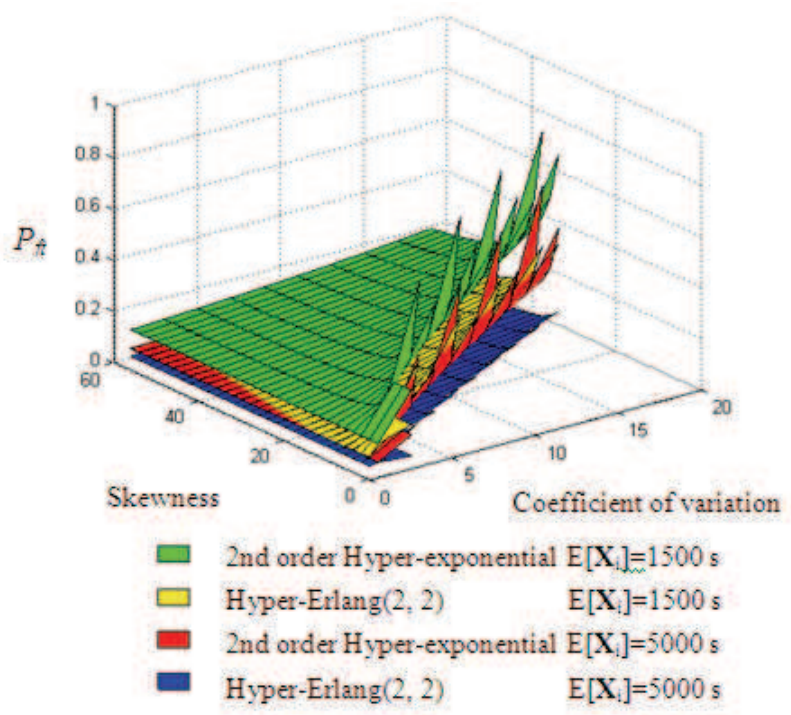

Fig. 3. Forced termination probability versus coefficient of variation and skewness of interruption time, with the pdf type and expected value of interruption time as parameters. 


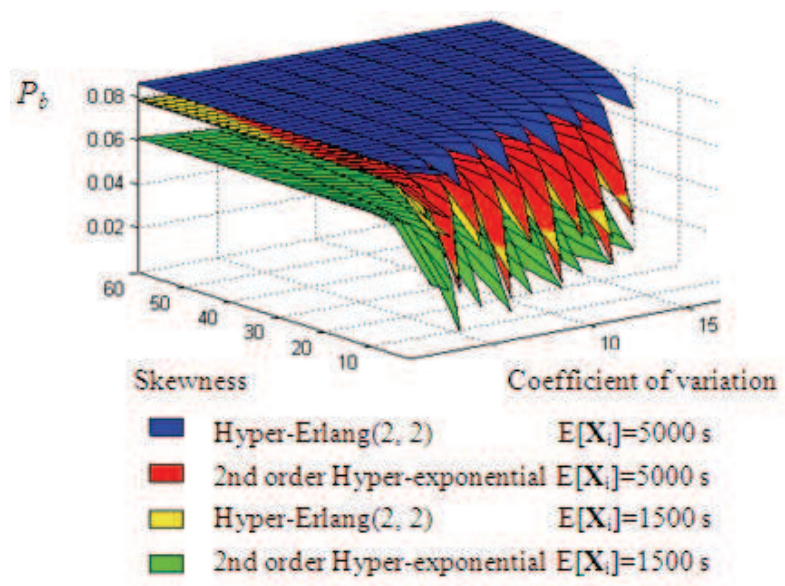

Fig. 4. New call blocking probability versus coefficient of variation and skewness of interruption time, with the pdf type and expected value of interruption time as parameters.

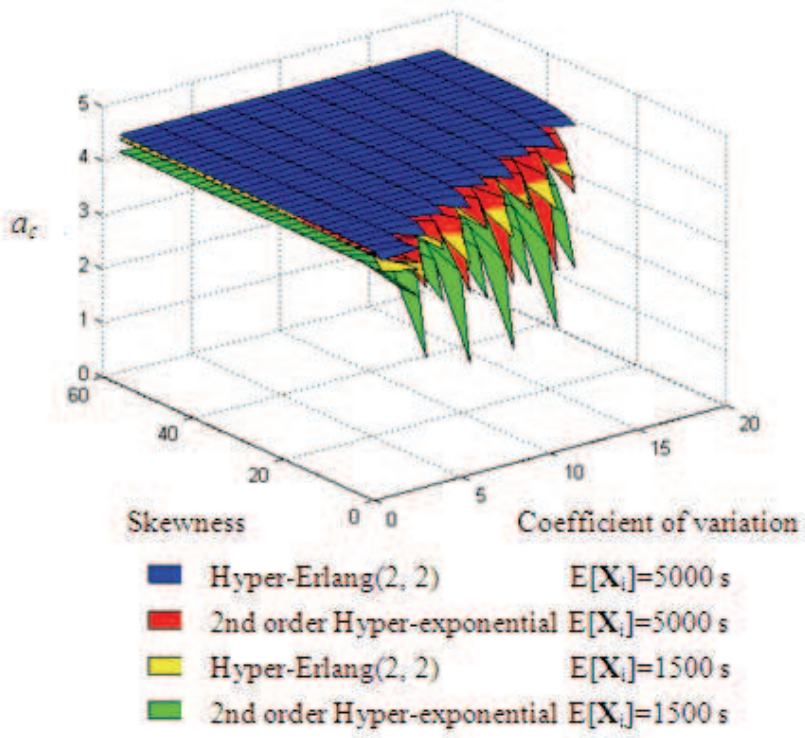

Fig. 5. Carried traffic versus coefficient of variation and skewness of interruption time, with the pdf type and expected value of interruption time as parameters.

\subsection{Influence of cell dwell time statistics on system performance}

In this Section, the influence of the expected value, coefficient of variation, and skewness of cell dwell time on system performance is investigated.

From Fig. 6 it is observed that as the mean value of the CDT decreases the forced termination probability increases, indicating a detrimental effect of mobility on system performance. This behavior can be explained by the fact that as the mean value of CDT 
decreases the average number of handoffs per call increases and, as consequence, the probability of a premature termination due to resource insufficiency increases. On the other hand, from Fig. 7, it is observed that the blocking probability increases as the mean value of CDT increases. This is because the larger the mean cell dwell time the slower users with ongoing calls move and, consequently, the rate at which radio resources are released decreases, causing a detrimental effect on blocking probability.

Fig. 6 also shows that, for the low mobility scenario, forced termination probability is practically insensitive to both skewness and CoV of CDT. This behavior is due to the fact that a low mobility scenario implies that most of the calls are completed (or blocked) in the cell where they were originated, reducing the average number of handoffs per call. Also, Fig. 6 shows that, for the high mobility scenario and irrespective of the value of skewness, forced termination probability decreases as $\mathrm{CoV}$ of CDT increases. This behavior can be explained as follows. First, note that as the $\mathrm{CoV}$ of CDT increases, the variability of the CDT increases, thus the probability that CDT takes higher values increases and, consequently, the average number of handoffs per call decreases, resulting in an improvement on the forced termination probability.

Furthermore, from Fig. 6 it is rather interesting to note that, for the high mobility scenario and low values of skewness (say, less that 20), forced termination probability is significantly improved as $\mathrm{CoV}$ of CDT increases. For instance, Fig. 6 shows that, for the high mobility scenario where CDT is Hyper-Erlang distributed, the forced termination probability decreases around $60 \%$ as the skewness and $\mathrm{CoV}$ of UIT change from 60 to 2 and from 1 to 20 , respectively. Again, notice that the scenario where skewness equals 2 and CoV equals 1 corresponds to the case when CDT is negative exponential distributed.

On the other hand, Figs. 6 and 7 show that, for the high mobility scenario both forced termination and new call blocking probabilities are monotonically increasing functions of skewness of CDT. The reason is as follows. Consider that the mean value, CoV and distribution type of CDT remain without change. Then, as the skewness of CDT decreases, the tail on the right side of the CDT distribution function becomes longer (that is, the probability that CDT takes higher values increases and, consequently, less calls move to

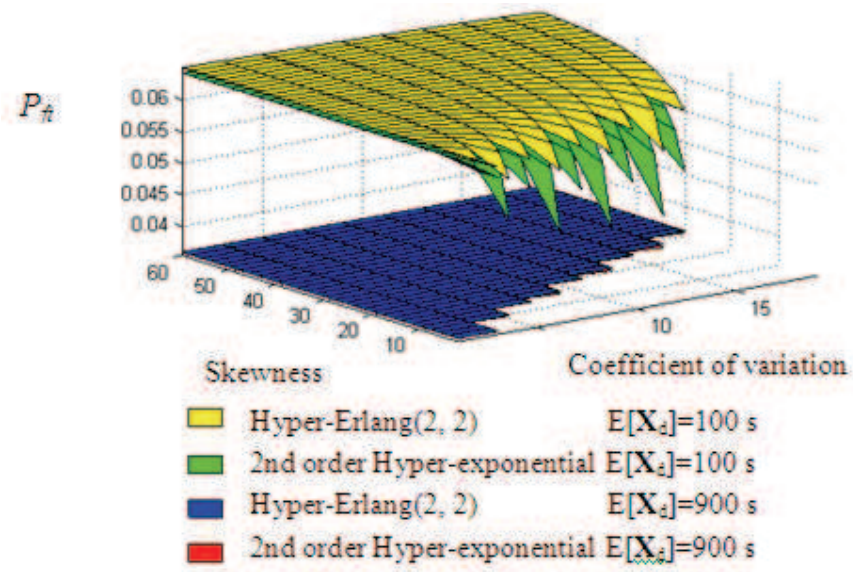

Fig. 6. Forced termination probability versus coefficient of variation and skewness of cell dwell time, with the pdf type and mean value of cell dwell time as parameters. 
another cell). In this manner, the rate at which channels are used by handed off calls decreases in benefit of both new call blocking probability and handoff failure probability (and, thus, forced termination probability). This fact contributes to improve carried traffic in concordance with the results presented in Fig. 8. Fig. 8 shows that carried traffic is a decreasing function of skewness of CDT. Also, Fig. 8 shows that carried traffic increases as $\mathrm{CoV}$ of CDT increases. These observations indicate a beneficial effect of the variability of CDT on carried traffic.

Finally, as it was observed in the previous section, the results illustrated in Figs. 6-8 show that, for the same scenario, skewness and $\mathrm{CoV}$ of CDT, there exists a non-negligible difference between the values taken by the different performance metrics when CDT is modeled as hyper-Erlang and hyper-exponential distributed random variable. Thus, it is again evident that not only the expected value but also moments of higher order and the distribution model used to characterize cell dwell time are relevant on system performance.

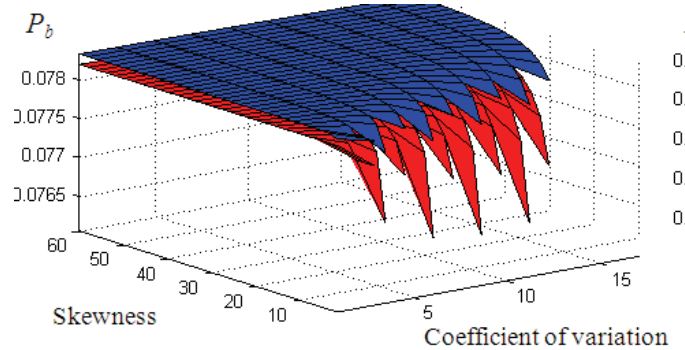

Hyper-Erlang $(2,2) \quad E\left[\mathbf{X}_{d}\right]=900 \mathrm{~s}$ 2nd order Hyper-exponential $\mathrm{E}\left[\mathbf{X}_{\mathrm{d}}\right]=900 \mathrm{~s}$

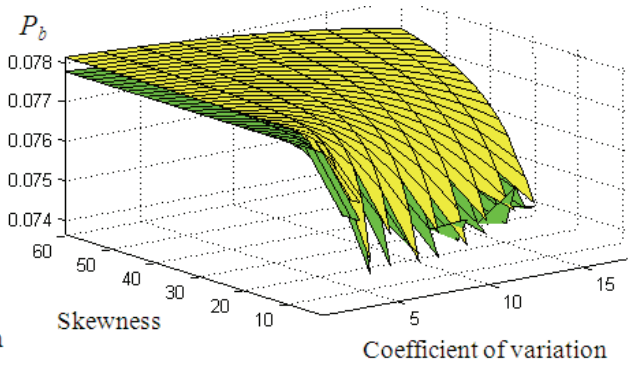

$\square$ Hyper-Erlang $(2,2) \quad \mathrm{E}\left[\mathbf{X}_{\mathrm{d}}\right]=100 \mathrm{~s}$ 2nd order Hyper-exponential $\mathrm{E}\left[\mathbf{X}_{\mathrm{d}}\right]=100 \mathrm{~s}$

Fig. 7. New call blocking probability versus coefficient of variation and skewness of cell dwell time, with the pdf type and expected value of cell dwell time as parameters.
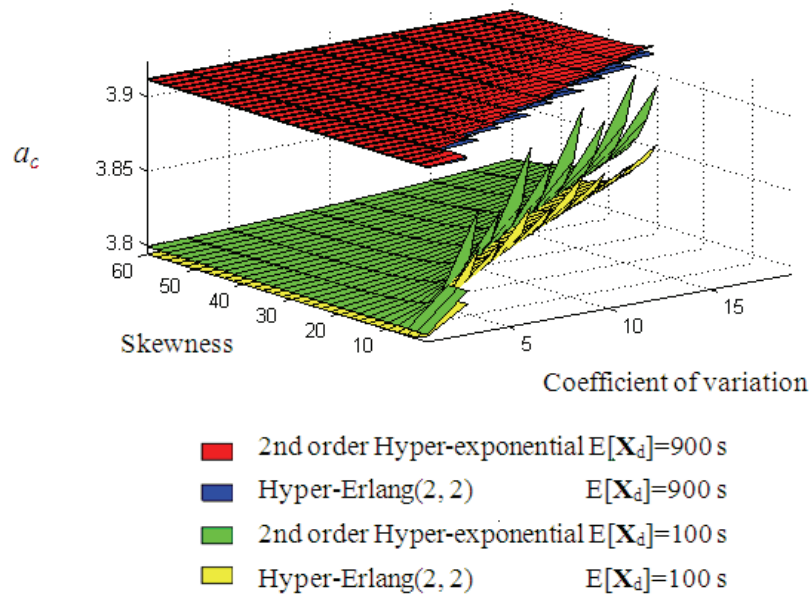

Fig. 8. Carried traffic versus coefficient of variation and skewness of cell dwell time, with the probability density function type and expected value of cell dwell time as parameters. 


\section{Conclusion}

The study performed in this Chapter have allowed us to obtain new and important insights into the dependence of system performance on the first three standardized moments of both cell dwell time and unencumbered interruption time. Even though our numerical results are extracted from particular scenarios with certain set of parameter values, our contribution clearly shows that there exist relevant sensitive issues concerning higher order moments of both cell dwell time and unencumbered interruption time. We conclude that to accurately characterize the real distribution of the different random variables involved in the teletraffic model it is vital to consider not only the mean value but also higher order moments.

\section{References}

Alfa A. S., and Li W., "A homogeneous PCS network with Markov call arrival process and phase type cell dwell time," Wireless Networks, vol. 8, no. 6, pp. 597-605, 2002.

G. Boggia, P. Camarda, A. D'Alconzo, A. De Biasi and M. Siviero, "Drop call probability in established cellular networks: from data analysis to modelling," in Proc. IEEE VTC'05-Spring, Stockholm, Sweden, vol. 5, May-Jun. 2005, pp. 2775-2779.

Christensen T. K., Nielsen B.F., and Iversen V.B., "Phase-type models of channel-holding times in cellular communication systems," IEEE Trans. Veh. Technol., vol. 53, no. 3, pp. 725-733, May 2004.

Cooper B., Introduction to Queuing Theory. CEE Press Books, Washington D.C. 1990.

Corral-Ruiz A.L.E., Cruz-Pérez F.A., and Hernández-Valdez G., "Teletraffic model for the performance evaluation of cellular networks with hyper-Erlang distributed cell dwell time," Proc. 71st IEEE VTC'10-Spring, Taipei, Taiwan, 16-19 May 2010.

Del Re E., Fantacci R., and Giambene G., "Handover and dynamic channel allocation techniques in mobile cellular networks," IEEE Trans. Veh. Technol., vol. 44, no. 2, pp. 229-237, 1995.

Fang Y., "Hyper-Erlang distributions of traffic modeling in wireless and mobile networks," in Proc. IEEE WCNC'99, New Orleans, LA, Sept. 1999, pp.398-402.

Fang Y., a, "Modeling and performance analysis for wireless mobile networks: a new analytical approach," IEEE Trans. on Networking, vol. 13,no. 5, pp. 989-1002, Oct. 2005.

Fang Y., b, "Performance evaluation of wireless cellular networks under more realistic assumptions," Wireless Commun. Mob. Comp., vol. 5, no. 8, pp. 867-885, Dec. 2005.

Fang Y. and Chlamtac I. "Teletraffic analysis and mobility modeling of PCS networks," IEEE Trans. Commun., vol 47, no. 7, pp. 1062-1072, July 1999.

Fang Y., Chlamtac I., and Lin Y.-B., a, "Call performance for a PCS network," IEEE J. Select. Areas Commun., vol. 15, no. 8, pp. 1568-1581, Oct. 1997.

Fang Y., Chlamtac I., and Lin Y.-B., b, "Modeling PCS networks under general call holding time and cell residence time distributions," IEEE/ACM Trans. on Networking, vol. 5, no. 6, pp. 893-906, Dec. 1997.

Guérin R., "Channel occupancy time distribution in a cellular radio system," IEEE Trans. Veh. Technol., vol. VT-35, pp. 89-99, Aug. 1987.

Hong D. and Rappaport S. S., "Traffic model and performance analysis for cellular mobile radio telephone systems with prioritized and nonprioritized handoff procedures," IEEE Trans. Veh. Technol., vol. 35, no. 3, pp. 77-92, Aug. 1986.

Khan F. and Zeghlache D., "Effect of cell residence time distribution on the performance of cellular mobile networks," in Proc. IEEE Veh. Tech. Conf.'97, Phoenix, AZ, 1997, pp. 949-953. 
Kim K. and Choi H., "A mobility model and performance analysis in wireless cellular network with general distribution and multi-cell model," Wireless Pers. Commun., published on line: 10 March 2009.

Li W. and Fang Y., "Performance evaluation of wireless cellular networks with mixed channel holding times," IEEE Trans. Wireless Communs., vol. 7, no. 6, June 2008.

Lin Y.-B., Mohan S. and Noerpel A., "Queuing priority channel assignment strategies for PCS and handoff initial access," IEEE Trans. Veh. Technol., vol. 43. no. 3, pp. 704-712, Aug. 1994.

Orlik P. V. and Rappaport S.S., "A model for teletraffic performance and channel holding time characterization in wireless cellular communication with general session and dwell time distributions," IEEE J. Select. Areas Commun., vol. 16, no. 5, pp. 788-803, June 1998.

Pattaramalai S., Aalo V.A., and Efthymoglou G.P., "Evaluation of call performance in cellular networks with generalized cell dwell time and call-holding time distributions in the presence of channel fading," IEEE Trans. Veh. Technol., vol. 58, no. 6, pp. 3002-3013, July 2009.

Rahman, M. M., Alfa A. S., “Computationally efficient method for analyzing guard channel schemes," Telecommunication Systems, vol. 41, pp. 1-11, published on line: 22 April 2009.

Rico-Páez A., Cruz-Pérez F.A., and Hernández-Valdez G., “Teletraffic Analysis Formulation based on Channel Holding Time Statistics," in Proc. IEEE International Conference on Wireless and Mobile Computing, Networking and Communications (WiMob'2009), Marrakech, Morocco, 12-14 October 2009.

Rico-Páez A., Cruz-Pérez F.A., and Hernández-Valdez G., "Performance Sensitivity to Higher Order Moments of Call Interruption and Cell Dwell Times in Cellular Networks," Proc. 72nd IEEE Vehicular Technology Conference (VTC'10-Fall), Ottawa, Canada, 6-9 September 2010.

Rico-Páez A., Rodríguez-Estrello C. B., Hernández-Valdez G., and Cruz-Pérez F. A., "Queueing Analysis of Mobile Cellular Networks Considering Wireless Channel Unreliability and Resource Insufficiency," Lecture Notes in Computer Science (LNCS 4516) -Managing Traffic Performance in Converged Networks-, L. Mason, T. Drwiega, and J. Yan (Editors): ITC20 2007, pp. 938-949, 2007.

Rodríguez-Estrello C. B., Hernández-Valdez G., and Cruz-Pérez F. A., "System level analysis of mobile cellular networks considering link unreliability," IEEE Trans. Veh. Technol., vol. 58, no. 2, pp. 926-940, Feb. 2009.

Rodríguez-Estrello C. B., Hernández-Valdez G., and Cruz-Pérez F. A., Chapter: "Performance Modeling and Analysis of Mobile Wireless Networks," for the book "Mobile and Wireless Communications Physical Layer Development and Implementation," InTech Publisher, Salma Ait Fares and Fumiyuki Adachi (Ed.). Published: January 2010.

Vázquez-Ávila J.L., Cruz-Pérez F.A., and Ortigoza-Guerrero L., "Performance analysis of fractional guard channel policies in mobile cellular networks" IEEE Trans. on Wireless Commun., vol. 5, no. 2, pp. 301-305, Feb. 2006.

Wang X. and Fan Pingzhi, "Channel holding time in wireless cellular communications with general distributed session time and dwell time," IEEE Communications Letters, vol. 11, no. 2, Feb. 2007.

Yeo K. and Jun C.-H., "Teletraffic analysis of cellular communication systems with general mobility based on hyper-Erlang characterization," Computer $\mathcal{E}$ Industrial Engineering, vol. 42, pp. 507-520, 2002.

Zeng H., Fang Y., and Chlamtac I., "Call blocking performance study for PCS Networks under more realistic mobility assumptions," Telecommun. Systems, vol. 19, no. 2, pp. 125-146, 2002. 


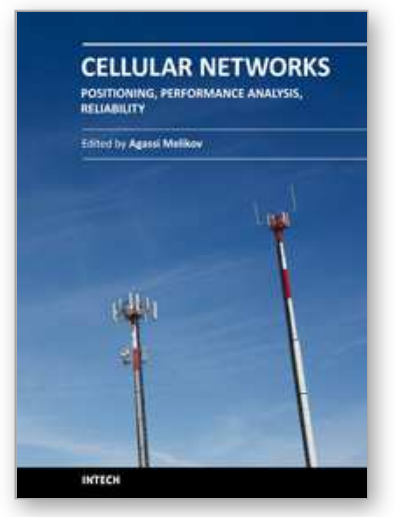

\author{
Cellular Networks - Positioning, Performance Analysis, Reliability \\ Edited by Dr. Agassi Melikov
}

ISBN 978-953-307-246-3

Hard cover, 404 pages

Publisher InTech

Published online 26, April, 2011

Published in print edition April, 2011

Wireless cellular networks are an integral part of modern telecommunication systems. Today it is hard to imagine our life without the use of such networks. Nevertheless, the development, implementation and operation of these networks require engineers and scientists to address a number of interrelated problems. Among them are the problem of choosing the proper geometric shape and dimensions of cells based on geographical location, finding the optimal location of cell base station, selection the scheme dividing the total net bandwidth between its cells, organization of the handover of a call between cells, information security and network reliability, and many others. The book focuses on three types of problems from the above list Positioning, Performance Analysis and Reliability. It contains three sections. The Section 1 is devoted to problems of Positioning and contains five chapters. The Section 2 contains eight Chapters which are devoted to quality of service (QoS) metrics analysis of wireless cellular networks. The Section 3 contains two Chapters and deal with reliability issues of wireless cellular networks. The book will be useful to researches in academia and industry and also to post-gradute students in telecommunication specialitiies.

\title{
How to reference
}

In order to correctly reference this scholarly work, feel free to copy and paste the following:

Felipe A. Cruz-Pérez, Genaro Hernández-Valdez and Andrés Rico-Páez (2011). Call-Level Performance Sensitivity in Cellular Networks, Cellular Networks - Positioning, Performance Analysis, Reliability, Dr. Agassi Melikov (Ed.), ISBN: 978-953-307-246-3, InTech, Available from: http://www.intechopen.com/books/cellularnetworks-positioning-performance-analysis-reliability/call-level-performance-sensitivity-in-cellular-networks

\section{INTECH}

open science | open minds

\section{InTech Europe}

University Campus STeP Ri

Slavka Krautzeka 83/A

51000 Rijeka, Croatia

Phone: +385 (51) 770447

Fax: +385 (51) 686166

www.intechopen.com

\section{InTech China}

Unit 405, Office Block, Hotel Equatorial Shanghai

No.65, Yan An Road (West), Shanghai, 200040, China 中国上海市延安西路65号上海国际贵都大饭店办公楼405单元

Phone: +86-21-62489820

Fax: +86-21-62489821 
(C) 2011 The Author(s). Licensee IntechOpen. This chapter is distributed under the terms of the Creative Commons Attribution-NonCommercialShareAlike-3.0 License, which permits use, distribution and reproduction for non-commercial purposes, provided the original is properly cited and derivative works building on this content are distributed under the same license. 